\title{
Evaluation of the Lactate Threshold during Downhill Running in Rats
}

\author{
Wakako Tsumiyama ${ }^{1,2)^{*}}$, Sadaaki Oki ${ }^{1)}$, Namiko Umeil ${ }^{1)}$, Michele Eisemann Shimizu ${ }^{3)}$, \\ TAKEYA ONO ${ }^{1)}$, AKIRA OTSUKA ${ }^{1)}$ \\ 1) Department of Physical Therapy, Faculty of Health and Welfare, Prefectural University of \\ Hiroshima: 1-1 Gakuen Machi, Mihara City, Hiroshima 723-0053, Japan \\ 2) Program in Biological System Sciences, Graduate School of Comprehensive Scientific Research, \\ Prefectural University of Hiroshima, Japan \\ 3) Department of Nursing and Rehabilitation, Konan Women's University, Japan
}

\begin{abstract}
Purpose] The purpose of the present study was to investigate if lactate thresholds in rats could be accurately determined during downhill running in rats. [Subjects] Twenty-four female Wistar rats were used in this study. Rats were randomly divided into two groups: the level running group and downhill running group. [Methods] Catheters were inserted into the right external jugular veins of the rats. Two days after the operation, the rats performed incremental exercise tests. The level running group ran on a treadmill at a $0^{\circ}$ incline; the downhill running group ran on a treadmill at a $-16^{\circ}$ incline. The lactate threshold was determined using the 2-line combination that minimizes the total sum of squares for fitting all data. [Results] Maximal running speeds and running speeds at the lactate threshold for the downhill running group were significantly higher than those for the level running group. [Conclusion] In this study, it was possible to accurately determine the lactate threshold during downhill running. These findings suggest that when the treadmill speed is the same, downhill running can be done at lower metabolic intensity than level running.

Key words: Lactate threshold, Downhill running, Eccentric contraction
\end{abstract}

(This article was submitted Jul. 8, 2013, and was accepted Aug. 17, 2013)

\section{INTRODUCTION}

To obtain hypertrophy of skeletal muscle, it is necessary to perform high-intensity training. However, it is difficult for the elderly to perform high-intensity training when they have decreased in cardiopulmonary function. Therefore, it is necessary to develop an effective low-intensity training program.

Eccentric contraction has been shown to produce greater muscle hypertrophy than concentric contraction after training $^{1,2)}$. In addition to greater hypertrophy, eccentric contraction training tends to exert a lower amount of stress on the cardiovascular system ${ }^{3}$. When eccentric contractions are used, lower-intensity training can be used than when concentric contractions are used to produce muscle hypertrophy. However, there are no reports on eccentric contraction training based on the lactate threshold in rats. In this study, we propose a protocol for accurate determination of the lactate threshold using eccentric contraction training in rats. Armstrong et al. ${ }^{4}$ reported on using eccentric

*Corresponding author. Wakako Tsumiyama (e-mail: tsumiyama@pu-hiroshima.ac.jp)

(C2014 The Society of Physical Therapy Science

This is an open-access article distributed under the terms of the Creative Commons Attribution Non-Commercial No Derivatives (by-ncnd) License $<$ http://creativecommons.org/licenses/by-nc-nd/3.0/> . contractions through downhill running in rats. They used downward movement patterns to stimulate the antigravity muscles through eccentric contractions. Downhill running is widely used as a method for eccentric contraction training of skeletal muscle in rats ${ }^{4-6)}$. Therefore, in this experiment, we used downhill running as the eccentric contraction training.

\section{SUBJECTS AND METHODS}

Twenty-four female Wistar rats (10-12 weeks of age, $241.8 \pm 14.5 \mathrm{~g}$ ) were used in this study. The rats were randomly divided into two groups of twelve rats each: the level running group and the downhill running group. The level running group ran on a treadmill at a $0^{\circ}$ incline. The downhill running group ran on a treadmill at a $-16^{\circ}$ incline. The rats were housed in a temperature-controlled room at $23{ }^{\circ} \mathrm{C}$ with a 12-hour light-dark cycle and were given free access to standard rat food and water. The protocols of this study were approved by the Animal Experiment Committee of the Prefectural University of Hiroshima (No. 12MA009).

The rats were anesthetized with diethyl ether, and a catheter was inserted into the right external jugular vein for blood sampling according to the method reported by Hashimoto et $\left.\mathrm{al}^{7}\right)$. Heparinized saline $(100 \mathrm{IU} / \mathrm{ml})$ was infused into the catheter to prevent blood coagulation. Two days after the operation, the rats performed incremental exercise tests. The exercise tests continued until the rat could no lon- 
Table 1. Blood lactate concentration and running speed during the incremental exercise test (mean \pm $\mathrm{SD}$ or median [minimum - maximum])

\begin{tabular}{lcc}
\hline & $\begin{array}{c}\text { Level running group } \\
(\mathrm{n}=12)\end{array}$ & $\begin{array}{c}\text { Downhill running group } \\
(\mathrm{n}=12)\end{array}$ \\
\hline Lactate at rest $(\mathrm{mmol} / \mathrm{L})$ & $2.28 \pm 0.86$ & $2.13 \pm 0.55$ \\
Lactate at maximal level $(\mathrm{mmol} / \mathrm{L})$ & $13.6 \pm 3.43$ & $14.4 \pm 2.50$ \\
Running speed at lactate threshold $(\mathrm{m} / \mathrm{min})$ & $27.9 \pm 4.68$ & $31.6 \pm 5.14^{*}$ \\
Running speed at maximal level $(\mathrm{m} / \mathrm{min})$ & $38.0(30.0-42.0)$ & $42.0(38.0-50.0)^{*}$ \\
\hline
\end{tabular}

$* \mathrm{p}<0.05$. Significant differences compared with the level running group

ger run. A treadmill made for rats and mice (Exer-3/6, Columbus Co., Ltd) was used. The initial speed of the treadmill was $10 \mathrm{~m} / \mathrm{min}$, and the treadmill speed was increased every 2 minutes by $2 \mathrm{~m} / \mathrm{min}^{8)}$. Blood samples $(5 \mu \mathrm{L})$ were taken from the jugular vein catheter at rest and every 2 minutes afterwards for analysis ${ }^{8)}$. Blood lactate concentrations were measured using a portable lactate analyzer (Lactate Pro; Arkray, Japan). The blood lactate endurance marker software (Lactate-E) ${ }^{9)}$ determined the 2-line combination that minimizes the total sum of squares for fitting all data. To determine the lactate threshold, a graph was made from the results of the blood lactate concentrations at the different running speeds.

Results are presented as mean \pm standard deviation or median (minimum-maximum) values. The unpaired t-test or the Mann-Whitney U test were used to compare the values of the level running group and downhill running group. Significance was considered to be $\mathrm{p}<0.05$.

\section{RESULTS}

Table 1 shows the blood lactate concentration and running speed during the incremental exercise test. There was no difference between the level running group and the downhill running group with regard to the results of the blood lactate concentration at rest and the maximal blood lactate concentration. The maximal running speeds were $38.0(30.0-42.0) \mathrm{m} / \mathrm{min}$ for the level running group and 42.0 (38.0-50.0) $\mathrm{m} / \mathrm{min}$ for the downhill running group. Maximal running speeds for downhill running were significantly higher than those for level running $(\mathrm{p}<0.05)$. The running speeds at the lactate threshold were $27.9 \pm 4.68 \mathrm{~m} / \mathrm{min}$ for the level running group and $31.6 \pm 5.14 \mathrm{~m} / \mathrm{min}$ for the downhill running group. Running speeds at the lactate threshold for downhill running were significantly higher those for than level running $(\mathrm{p}<0.05)$.

\section{DISCUSSION}

The current study evaluated the lactate threshold during level and downhill running in rats. The authors were able to accurately determine the lactate threshold of rats by taking sequential blood samples during incremental exercise tests.

As shown in Table 1, the maximal lactate concentrations and those at rest were not significantly different between downhill running and level running. However, the maximal running speed and the running speed at the lactate threshold significantly increased during downhill running as compared with level running. These data may then help show that when the treadmill speed is the same, downhill running can be done at a lower metabolic intensity than when level running is done. In other words, downhill running tend to exert a lower amount of stress on the cardiovascular system. Previous reports ${ }^{10,11)}$ showed that the maximal oxygen uptake and heart rate decreased more during downhill running as compared with level running and uphill running. Previous reports ${ }^{2)}$ also showed that, as compared with concentric contractions, eccentric contractions are more effective in increasing muscle strength and hypertrophy. When the exercise intensity is the same, rather than using level running, downhill running may be a good choice for increasing muscle strength and hypertrophy. These findings suggest that downhill training may be effective for training of elderly. However, safety monitoring is important because there is the possibility of falling and injury.

In future studies, we will attempt to do further research on effective training for inducing hypertrophy of skeletal muscle via low-intensity downhill training in rats.

\section{REFERENCES}

1) Enoka RM: Eccentric contractions require unique activation strategies by the nervous system. J Appl Physiol, 1996, 81: 2339-2346. [Medline]

2) Farthing JP, Chilibeck PD: The effect of eccentric and concentric training at different velocities on muscle hypertrophy. Eur J Appl Physiol, 2003, 89: 578-586. [Medline] [CrossRef]

3) Okamoto T, Masuhara M, Ikuta K: Cardiovascular responses induced during high intensity eccentric and concentric isokinetic muscle contraction in healthy young adults. Clin Physiol Funct Imaging, 2006, 26: 39-44. [Medline] [CrossRef]

4) Armstrong RB, Ogilvie RW, Schwane JA: Eccentric exercise-induced injury to rat skeletal muscle. J Appl Physiol, 1983, 54: 80-93. [Medline]

5) Matsubara T, Miki A: Effects of stretch on muscle regeneration in the damaged mouse soleus muscle after eccentric exercise. Bull Health Sci Kobe, 2005, 21: 1-11.

6) Shima Y, Kitaoka K, Yoshiki Y, et al.: Effect of heat shock preconditioning ROS scavenging activity in rat skeletal muscle after downhill running. J Physiol Sci, 2008, 58: 341-348. [Medline] [CrossRef]

7) Hashimoto H, Saito T, Takahashi K, et al: A new method of jugular vein cannulation for blood samples in rats. Journal of Experimental Animal Technology, 2001, 36: 1-8 (in Japanese).

8) Tsumiyama W, Oki S, Tamaru M, et al.: Evaluation of the lactate threshold of rats using external jugular vein catheterization. J Phys Ther Sci, 2012, 24: 1107-1109. [CrossRef]

9) Newell J, Higgins D, Madden N, et al.: Software for calculating blood lactate endurance markers. J Sports Sci, 2007, 25: 1403-1409. [Medline] [CrossRef]

10) Abe D, Fukuoka Y, Muraki S, et al.: Effects of load and gradient on energy cost of running. J Physiol Anthropol, 2011, 30: 153-160. [Medline] [CrossRef]

11) Sasaki M, Yamagami H, Shiratori $T$ : The work road during downhill walking on treadmill. J Jpn Phys Ther Assoc, 2000, 27: 17-23 (in Japanese). 